\title{
A Comparative Analysis of Retinal Blood Vessels Approaches
}

\author{
Karthika.D \\ Ph.D., Research Scholar, Karpagam University, \\ Coimbatore Tamil Nadu, India.
}

\begin{abstract}
Image processing is intended for which the input is an image, like photograph or some other videos the output of may be whichever an image or, a set of characteristics or limitations consistent to the image. Retinal images has numerous qualitative procedures can be used in more applications, such as ocular fundus operations with human recognition. Likewise, it plays important roles in detection of a few diseases in premature stages, such as diabetes, which can be performed by evaluation of the states of retinal blood vessels. Inherent characteristics of retinal images make the blood vessel detection process tricky. This survey presents an analysis of several algorithms proposed by various authors to detect the retinal blood vessels effectively. The existing algorithms are analyzed thoroughly to identify their advantages and limitations. The performance evaluation of the existing algorithms is carried out to determine the best approach. Then, in order to improve the performance of the best approach, a novel approach is been proposed in this paper.
\end{abstract}

\section{Keywords}

Retina, fundus image, blood vessels, Curvelet, Contourlet, Diabetic retinopathy

\section{INTRODUCTION}

Retinal image processing is an important tool in diagnosing and curing the diseases that influence the retina and the choroid [1]. Diabetic retinopathy is the problem of diabetes mellitus pretends the retina and the choroid. In this scenario, a network of small blood vessels, called Choroidal Neovascularization (CNV), take place in the choroid and enchanting a part of the blood supplying the retina [1]. At the same time as the quantity of blood supplying to the retina is decreased, the view may be ruined and in the harsh cases, blindness may happen. The doctor attempts to treat this risky disorder by giving optical energy to photocoagulate the neovascularization. Laser Argon is used in photocoagulation to close off the part of it in a process of the small vessels which increase the quantity of blood supplying to the retina and so preserving the vision of the eye (Mai S. Mabrouk et al, 2006). This action modality is attained in numerous sessions. The medical doctor enquired the patient to possess his or her eye capable to direct the laser beam to the pretentious area. The present achievement rate of this process is below $50 \%$ for annihilation of $\mathrm{CNV}$ subsequent to one treatment session with a reappearance and/or persistence rate of about $50 \%$ [2].

\author{
Marimuthu.A,Ph.D \\ Associate Professor, Dept. of Computer Science, \\ Government Arts College, Tamil Nadu, India. \\ $\mathrm{m}$
}

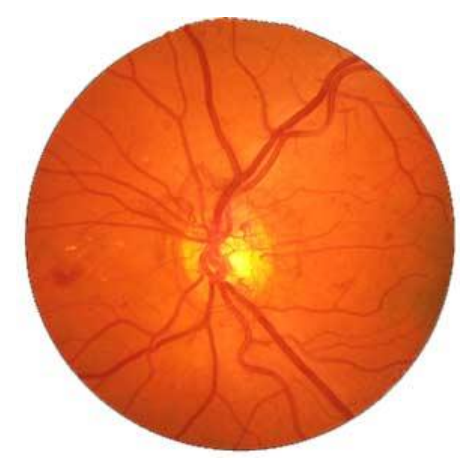

Fig 1: An Example of Retinal Blood Vessel

The last condition needs to replicate the treatment action. Each treatment replication has $50 \%$ failure rate. More than a few studies point out that incomplete treatment was linked with not as good as prognosis than no treatment. As a result, there is a need to build up an automated laser system to care for the entire retina in single session has become a necessity [1]. This system is planned to scan the retina and footpath it applying the laser energy to entire area apart from the sensitive objects that may be injured by the laser energy. The system is understood to do this by capturing the retinal images by means of a fundus camera. These images are segmented to take out the sensitive objects in the retina such as the blood vessel tree, the optic disk, the macula and the area flanked by the optic disk and the macula. The location of laser shots is to be spread in the rest of the retina. In addition a robust registration method is to be functional to spot the motion parameters of the retina to revise the positions of laser shots.

Retinal blood vessel changes have been reported to be associated with a number of diseases [3]. A large number of techniques have been developed for the analysis of retinal blood vessels. This paper aims to investigate the existing techniques available in the literature for analysis of retinal blood vessels. The problems and limitations of the existing techniques are evaluated and a comparative study is presented. Based on the comparative results, a better and suitable approach in retinal blood vessel analysis is also suggested in this paper.

\section{SURVEY ON RETINAL IMAGE TECHNIQUES}

Segmentation plays a primary role in a vast number of image processing applications. Majority of attention has been focused on segmentation of gray-level images as these have been the only kind of visual information. In recent years, color imagery has displaced monochromatic information and computation power and is no longer a limitation in processing huge volumes of data. Luccheseyz and Mitray [4] provided a review of techniques advanced in the past decades for segmentation of color images. 
Retinal image related diseases and its diagnosis has attracted various researchers due to the evolution of color image segmentation.

Sinthanayothin et al. [5] have previously discussed about several approaches employed in image pre-processing and identification of the optic disc, fovea and retinal blood vessels. This investigation has extended to the earlier work by pointing out that it is feasible to use recursive region growing segmentation approaches to identify hard exudates, haemorrhages and microaneurysms. A novel algorithm, 'Moat Operator', was utilized to optimize recognition of haemorrhages and microaneurysms. Recognition of hard exudates was high, with only faint exudates not determined. The recognition of haemorrhages and microaneurysms was a tough task. Additional works are needed to enhance the detection accuracy of these redcoloured lesions, which resembles with segments of small retinal blood vessels. Moreover, haemorrhages adjacent to blood vessels were also not identified by the algorithm. The correct evaluation of pathologies using the smallest sampling areas was not possible, so $10 \times 10$ pixel grids were overlaid upon the original image for the principle of localizing lesions. Each of the squares on the grid constituted a sampling unit. The amount of control regions of normal retina was high as only small regions of the image are exaggerated by pathology in most diabetic retina. This technique gives good results in recognition of necessary features of background diabetic retinopathy.

The Iterative Closest Point (ICP) registration algorithm is described and builds with recording retinal images. The ICP works under the process of iteration, there are two steps namely matching points based on the current transformation approximation and refining the approximation based on the matches. Charles V. Stewart et al [6] needs a good initial approximation. Other than, the Dual-Bootstrap ICP algorithm merely requires an initial evaluation that is a "toe hold" on the exact alignment over a small image region and perhaps by means of a lower-order transformation that is needed to align accurately the entire images. The datasets containing about 6000 image pairs, whereas it is effectively registered $99.5 \%$ of the pairs which are surrounded at least by one common landmark and $100 \%$ of the pairs containing at least one common landmark and $35 \%$ overlap or higher. This technique is close to perfect performance enables different applications.

The above discussed approaches had several drawbacks such as lower PSNR and higher MSE. But, these approaches are considered as the fundamental basis for various retinal blood vessel techniques developed in the later years.

In [7] a computerized segmentation method is established by the author to segment the vessels in the retina. This method is used to viewing the retinal images automatically for diabetic retinopathy. It separates edges of the image with the vessel centerlines of the retina. The ridges produce primitives throughout line elements. Based on these line elements, an image is segmented into patches by allotting each image pixel to the closest line element. Every line element comprises of a local coordinate frame for its equivalent patch. For every pixel, feature vectors are evaluated that utilizes the attributes of the patches and the line fundamentals. The feature vectors are categorized through a NN-classifier and sequential forward feature selection. This method was estimated on a database consist of 40 physically labeled images. The method obtains a region beneath the receiver operating characteristic curve of 0.952 . This method is compared with rule-based methods of Hoover et al. (2000) [8] and Jiang et al. (2003) [9] and it is experiential that this method gives enhanced results than two rule-based methods $(\mathrm{p}<0.01)$.
In [10] the author studied about several performances of segmentation techniques of vessels in the retina. This approach is used to determine the infection of the eye. This approach is experimented using database of the screening images. The database is created by collection of 40 retinal images, the images contains vessel trees that are segmented physically to measure the performance of the system. The second self-governing physical segmentation is carried out for 20 of those 40 images. This helps to evaluate the performance of the automatic approaches. The experimental result shows that the author Zana et al [11] arithmetical morphology and curve estimation method, Jiang et al [12] verification-based local thresholding method and Chaudhuri et al [13] matched filter technique gives maximum average accuracy of $0.9377,0.9212$ and 0.8773 correspondingly. However, the pixel classification procedure is more efficient than the above methods and gives a maximum average accuracy of 0.9416 .

The retinal microvasculature is singular in that it is merely a part of the human circulation that can be visualized directly noninvasively in vivo, eagerly photographed and subject to digital image analysis. Exhilarating growth in image processing applicable to ophthalmology more than the past 15 years includes the advancement being completed towards developing automated diagnostic systems for conditions, like diabetic retinopathy, agerelated macular deterioration and retinopathy of prematurity. These diagnostic systems present here is possible to be used in large-scale screening programs, with the probable for important resource savings, in addition to being free from observer bias and exhaustion. Additionally, quantitative capacity of retinal vascular topography by means of digital image analysis from retinal photography. It has been used as explore tools to get more knowledge about the connection between the retinal microvasculature and cardiovascular disease. Niall Patton et al [14] give explanation about the principles based upon which retinal digital image analysis. The author said that the present technique automatically identify landmark features of the fundus, like optic disc, fovea and blood vessels. The author analyze about the usage of image analysis in diagnosis of pathology automatically.

Richard J. Radke et al [15] have endeavor with the current state of the art in image change detection without highlighting a specific application region. To facilitate classification of algorithms into a fairly small number of groups will give helpful assistance to the algorithm designer. The author experiential that there are some software packages that comprises some of the transform detection algorithms talk about here. Especially, the author has implemented a friendly website to this paper that contains implementations, results and comparative analysis of other methods discussed above. The author not tried to editorialize about the relative merits of the algorithms, leaving the reader to choose which supposition and restriction may be most applicable for his or her application. Though, the authors view is that two algorithms earn unique state. Background modeling algorithm, offer for multiple models of background look in addition to object look and movement. The author projected that a grouping of these approaches that uses soft assignment of each pixel to an object class and one or more change models would make a very wide-ranging, influential and tentatively well-justified change detection algorithm. In spite of the significant quantity of work in the field, change detection is still a dynamic and attractive area of research. Richard J. Radke et al [15] suggested that more integrated approaches combining elaborate models of implicit pre and post processing, robust statistics and global optimization techniques have to be developed. 
Mendonca and Campilho [16] presented an automated technique for the segmentation of the vascular network in retinal images. This algorithm initiates with the elimination of vessel centerlines, which are used as approach for the consequent vessel filling phase. Thus, the outputs of four directional differential operators are processed to select associated group of candidate points to be classified as centerline pixels via vessel derived features. An iterative region growing technique is used to get the finalized segmentation that incorporates the contents of a number of binary images resulting from vessel width dependent morphological filters. The results indicated that this approach outperforms other solutions and approximates the average accuracy of a human observer without a considerable deprivation of sensitivity and specificity.

The morphology of the retinal blood vessels can be an essential pointer diabetes, hypertension and Retinopathy of Prematurity (ROP). Thus, the evaluation of alterations in morphology of arterioles and venules can be of diagnostic value. Martinez-Perez et al [17] presented an approach to automatically segment retinal blood vessels based on multiscale feature extraction technique. This approach deals with the issues of differences in contrast inherent in the images through the initial and second spatial derivatives of the intensity image that offers data about vessel topology. This technique also facilitates the determination of blood vessels of different widths, lengths and orientations. The development gradually partitions the blood vessels through feature information connected with spatial information. The algorithm is evaluated on red-free and fluorescein retinal images. The results indicated that the approach attained $75.05 \%$ True Positive Rate (TPR) and 4.38\% False Positive Rate (FPR) from the first database where as the second database yields $72.46 \%$ TPR and $3.45 \%$ FPR. Alternatively, these values are not susceptible to assess the performance of vessel geometry detection. Thus, a novel technique that utilizes measurements of vessel diameters and branching angles as a validation measure to compare the segmented images with those hand segmented from public databases. The results showed that borders identified by this approach are less biased and resulted in good performance.

Mohammad Saleh Miri and Ali Mahloojifar [18] introduced a new algorithm to recognize the retinal blood vessels efficiently. Because of higher capability of the curvelet transform in denoting the edges, modification of curvelet transform coefficients to improve the retinal image edges better organizes the image for the segmentation component. The directionality feature of the multistructure elements technique makes it an effectual tool in edge detection. Thus, morphology operators through multistructure elements are known to the better image to decide the retinal image ridges. Then, morphological operators by reconstruction eradicate the ridges not involved to the vessel tree while trying to conserve the thin vessels unaltered. The consequence of the morphological operators by reconstruction is improved via the application of multistructure elements. An effortless thresholding method all along with Connected Components Analysis (CCA) shows the remained ridges fit in to vessels. In order to make use of CCA more capably, CCA and length filtering is applied relatively than taking into account the whole image. The experimental results in DRIVE database attains more than $94 \%$ accuracy in about $50 \mathrm{~s}$ for blood vessel detection, confirm that the blood vessels can be efficiently detected by applying this technique on the retinal images.

The initial enhancement of the image is done through Adaptive Histogram Equalization and then curvelet Transforms are applied to the equalized image and the curvelet coefficients are obtained [19]. The alterations to the Curvelet transform coefficients are done through suppressing all the coefficients of one band. The integrated form of the equalization and the Curvelet Transforms [20] offer a better enhancement to the image. This improved image is used for the extraction of the blood vessels and thresholding approach is utilized for the extraction of vessel. It comprises of spatial filtering of the image through the templates in eight different orientations. The masking of redundant regions in the output image is done through boundary approaches. The salt and pepper noise in the image are eliminated through median filtering to the image. Experiments were carried with a set of 20 images and have been proved that the algorithm provides good results for all the images.

Discrete Ripplet transform is used for enhancing the DRIVE database image [21]. It is observed that certain characteristics of retinal images make the vessel detection more difficult, therefore by performing enhancement before segmentation make the image appropriate for the segmentation process. The enhanced image is segmented through morphological operators. Morphological reconstruction is done to the segmented image so as to get the connected components of the image thus getting the reconstructed image.

The presence of hemorrhages in the retina is the earliest symptom of diabetic retinopathy. The number and shape of hemorrhages is used to indicate the severity of the disease. Prior automated detection of hemorrhage reduces the risk of blindness. Parisut Jitpakdee et al [22] reviewed methodologies that are used for the detection of hemorrhage from diabetic retinopathy retinal images. The automatic detection of the hemorrhages is a tough task. The hemorrhages are very difficult to differentiate from background variations as it typically low contrast.

By analyzing the retinal blood vessels, primary stages of diabetic retinopathy, age related macular degeneration, glaucoma etc can be diagnosed at the earlier stage. The main aim is to develop an efficient algorithm that segments the retinal blood vessels in a short time and with high accuracy. Sifna N Shajahan and Rajesh Cherian Roy [23] presented Curvelet transform for retinal image contrast enhancement. Multistructure elements morphology is used to identify the blood vessel edges as they are randomly distributed. The false edges are removed by morphological reconstruction. A locally applied level dependent thresholding algorithm with connected component analysis and length filtering removes the remaining false edges after reconstruction step. This algorithm is experimented with the images from the DRIVE database and it gave accuracy of about $97 \%$ in less than $15 \mathrm{~s}$, thus showing its effectiveness in retinal blood vessel segmentation. The Curvelet transform effectively denotes images containing edges with improved the image contrast for segmentation. The false edges are removed by morphological reconstruction, while preserving thin vessels. The application of Connected Component Analysis (CCA) and length filtering helped to eliminate the remaining false edges. In order to handle severe lesions in retinal images and also to improve the accuracy of this approach, better and efficient level dependent thresholding algorithm can be used.

Farnoosh Ghadiri et al [24] introduced a Non-sub sampled Contourlet Transform (NSCT) and the morphological operations for detection of images of the retina. The scales of the Contourlet image and the gray scale image are combined together to eradicate the vessel map. Optic disc edge is eliminated by Nonsub sampled Contourlet directional data. Moreover, circular shapes such as micro aneurysms are eliminated through morphological operations. Farnoosh Ghadiri et al (2013) examined this algorithm on retinal images of DRIVE database and conjuntival images of Khatam database. Experimental results showed significant improvements with high accuracy and decreasing False Positive Rate (FPR) of vessel detection on both 
databases. Moreover, simplicity and low time complexity are the other benefits of this vessel extraction technique.

\section{COMPARATIVE ANALYSIS OF THE EXISTING APPROACHES}

The performance evaluation of the above discussed approaches is based on certain performance metrics. The image enhancement performance metrics used in this research work are PSNR and MSE. The data set used for this experimental is the DRIVE dataset. The segmentation assessment is evaluated through three measures namely True Positive Rate (TPR), False Positive Rate (FPR), and Accuracy (ACC). It is denoted that TP and TN illustrate the blood vessel pixels and background pixels, which properly identified. FP demonstrates that the pixels are not fit in to a vessel, but is known as blood vessel pixels and FN shows the pixels belonging to a vessel, but is recognized as background pixels, incorrectly TPR, FPR, and Accuracy are defined as follows:

$$
\begin{gathered}
T P R=\frac{T P}{T P+F N} \\
F P R=\frac{F P}{F P+T N} \\
A C C=\frac{(T P+T N)}{T P+F P+T N+F N}
\end{gathered}
$$

Table 1. Contrast Enhancement Method Quantitative Assessment

\begin{tabular}{|c|c|c|c|}
\hline Approaches & TPR & FPR & $\begin{array}{c}\text { Average } \\
\text { Accuracy }\end{array}$ \\
\hline Staal et al, (2004) [7] & 0.6780 & 0.0170 & 0.9441 \\
\hline $\begin{array}{c}\text { Niemeijer et al } \\
\text { (2004) [10] }\end{array}$ & 0.6898 & 0.0304 & 0.9416 \\
\hline $\begin{array}{c}\text { Richard J. Radke et } \\
\text { al (2005) [15] }\end{array}$ & 0.6964 & 0.0300 & 0.9427 \\
\hline $\begin{array}{c}\text { Niall Patton et al } \\
\text { (2006) [14] }\end{array}$ & 0.7013 & 0.0288 & 0.9138 \\
\hline $\begin{array}{c}\text { Martinez-Perez et al } \\
\text { (2007) [17] }\end{array}$ & 0.7246 & 0.0345 & 0.9344 \\
\hline $\begin{array}{c}\text { Mohammad Saleh } \\
\text { Miri and Ali } \\
\text { Mahloojifar (2011) } \\
\text { [18] }\end{array}$ & 0.7352 & 0.0205 & 0.9458 \\
\hline $\begin{array}{c}\text { Campilho (2006) } \\
\text { [16] }\end{array}$ & 0.7344 & 0.0236 & 0.9452 \\
\hline $\begin{array}{c}\text { Performand ance C } \\
\text { Mendong }\end{array}$ & & & \\
\hline
\end{tabular}

\subsection{Performance Comparison}

Figure 2 shows the comparison of the TPR values of various approaches.

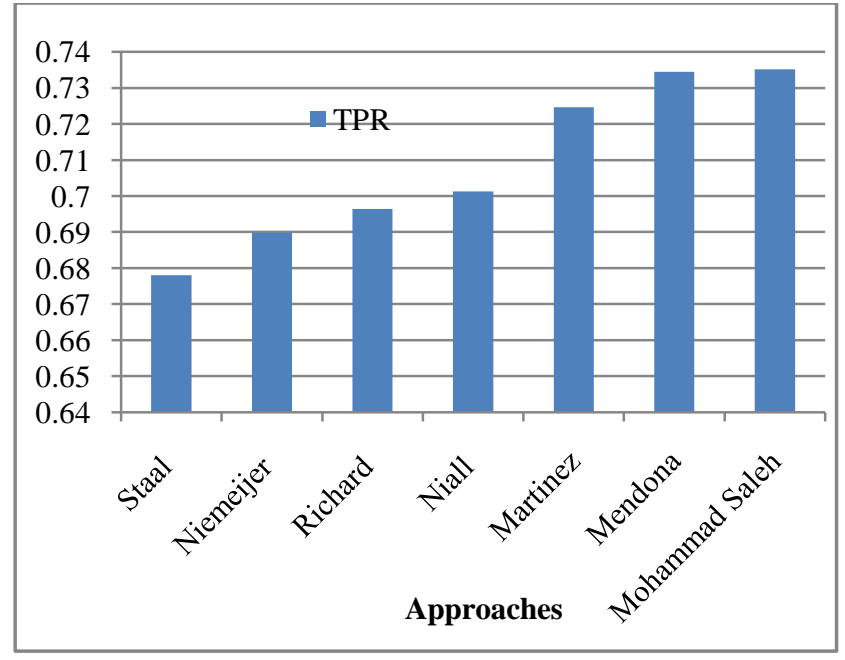

Fig 2: Comparison of TPR

It is observed from the figure that the approach by Mohammad Saleh Miri and Ali Mahloojifar (2011) [18] outperformed the other approaches in terms of TPR values. For instance, the TPR values by the Mohammad Saleh Miri and Ali Mahloojifar [18] approach is 0.7352 where as it is very lesser for the other approaches taken for consideration. The next best result is provided by Mendonca and Campilho (2006) [16] approach and Martinez-Perez et al (2007) approach [17] with values of 0.7344 and 0.7246 respectively.

Figure 3 shows the comparison of the FPR values for various existing approaches. It is observed from the graph that the Mohammad Saleh Miri and Ali Mahloojifar (2011) [18] approach outperformed the other approaches in terms of FPR.

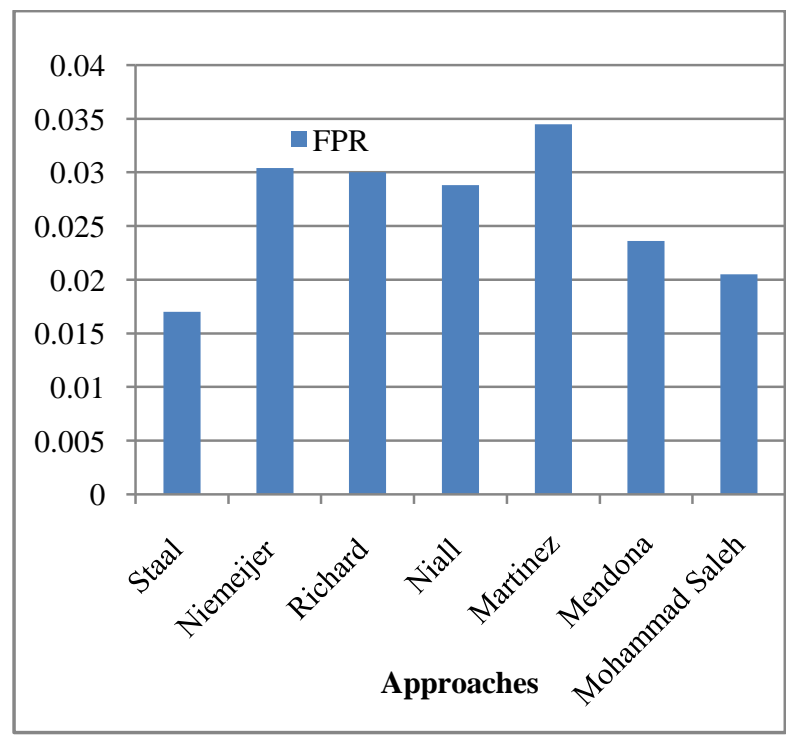

Fig 3: Comparison of FPR

Figure 4 shows the average accuracy of various existing approaches. The average accuracy of the Mohammad Saleh Miri and Ali Mahloojifar (2011) [18] approach is 0.9458 where as the other approaches is observed to provide lesser average accuracy. 


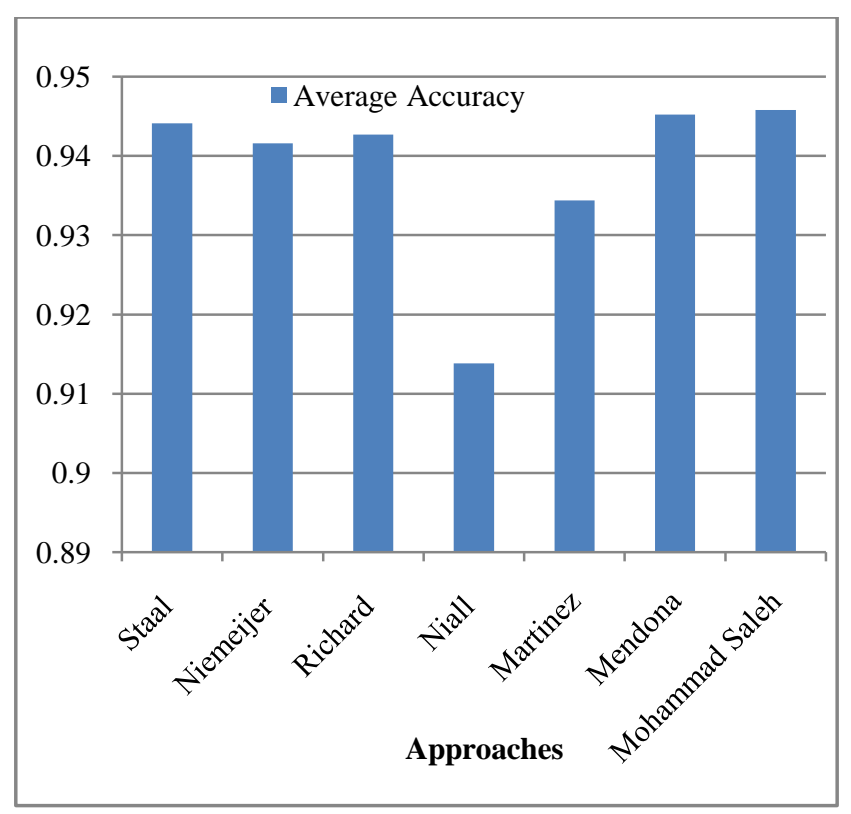

Fig 4: Comparison of Average Accuracy

It is inferred from the above results that the Mohammad Saleh Miri and Ali Mahloojifar (2011) approach which uses curvelet transformation provides significant results. Thus, the curvelet transformation has become an active research area in field of retinal image analysis.

\subsection{Performance evaluation of Curvelet Transformation}

The performance of the transformation techniques such as curvelt, Wavelet based curvelet and contourlet have been evaluated. Table 2 shows the PSNR values of the transform techniques taken for consideration for Drive database. It is observed from the table that the contourlet transformation provides better results than the curvelet transformation.

Table 2. Performance comparison of curvelet and contourlet based on PSNR and MSE values

\begin{tabular}{|c|c|c|}
\hline Approaches & PSNR & MSE \\
\hline $\begin{array}{c}\text { Curvelet } \\
\text { Transform }\end{array}$ & 28.53 & 0.934 \\
\hline $\begin{array}{c}\text { Contourlet } \\
\text { Transform }\end{array}$ & 30.39 & 0.810 \\
\hline $\begin{array}{c}\text { Wavelet based } \\
\text { Contourlet } \\
\text { Transform }\end{array}$ & 32.64 & 0.726 \\
\hline
\end{tabular}

\section{INFERENCE FROM THE EXISTING WORK}

The various transforms are wavelet transform, curvelet transform, contourlet transform and techniques are presented here. The shortcoming of wavelet transform is that there is a problem of filling missing data will take place and it has deprived directional specificity of the images [25]. In curvelet transform the image improvement cannot capture the geometric information of images and be liable to amplify noises when they are applied to noisy images also that they cannot distinguish noises from weak edges. The entire drawback is overcome by the Contourlet transform. Thus, Contourlet transform is considered as a novel research paradigm in this regards and efforts are made to improve the performance of the overall system through better transformation approaches.

\section{CONCLUSION}

In this paper a variety of retinal image analysis techniques are examined. It is clearly observed from the performance evaluation that the curvelet based approach for retinal blood vessel analysis provides significant results when compared with other existing techniques. But, curvelet transformation has certain drawbacks and Contourlet transform is a recently developed technique which can be more accurate and it overcomes all the problem of the other existing transformation techniques. Hence, contourlet transformation based retinal blood vessel analysis would offer significant results. Advance study includes growth of efficient algorithm to reduce computational cost and to decrease the time required for analyzing the image. Taking this as a motivation the contourlet transform is to be taken for the further research in order to improve the overall performance of the system.

\section{REFERENCES}

[1] Mai S. Mabrouk, Nahed H. Solouma and Yasser M. Kadah, "Survey of Retinal Image Segmentation and Registration", GVIP Journal, Volume 6, Issue 2, September, 2006.

[2] Nahed H. Solouma, Abou-Bakr M. Youssef, Yehia A. Badr, and Yasser M. Kadah, "Robust Computer-Assisted Laser Treatment Using Real- time Retinal Tracking”, 2001.

[3] Xiayu Xu, "Automated delineation and quantitative analysis of blood vessels in retinal fundus image", 2012.

[4] L. Luccheseyz and S.K. Mitray, "Color Image Segmentation: A State-of-the-Art Survey”, PINSA, pp. 207221, 2001.

[5] C. Sinthanayothin, J. F. Boyce, T. H. Williamson, H. L. Cook, E. Mensah, S. Lal and D. Usher, "Automated detection of diabetic retinopathy on digital fundus images" 2001.

[6] Charles V. Stewart, Chia-Ling Tsai, Badrinath Roysam, "The Dual-Bootstrap Iterative Closest Point Algorithm with Application to Retinal Image Registration", International Journal of Computer Vision June, 2002.

[7] J. Staal, M. D. Abramoff, M. Niemeijer, M. A. Viergever, and B. van Ginneken, "Ridge based vessel segmentation in color images of the retina," IEEE Trans. Med. Imag., vol. 23, no. 4, pp. 501-509, Apr. 2004.

[8] A. Hoover, V. Kouznetsova, and M. Goldbaum, "Locating blood vessels in retinal images by piecewise threshold probing of a matched filter response," IEEE Trans. Med. Imag., vol. 19, pp. 203-210, Mar. 2000.

[9] X. Jiang and D. Mojon, "Adaptive local thresholding by verification-based multithreshold probing with application to vessel detection in retinal images," IEEE Trans. Pattern Anal. Machine Intell., vol. 25, pp. 131-137, Jan. 2003.

[10] M. Niemeijer, J. Staal, B. van Ginneken, M. Loog, and M. D. Abr'amoff, "Comparative study of retinal vessel segmentation methods on a new publicly available database," Proc. SPIE-Med. Imag., vol. 5370, pp. 648656, 2004.

[11] F. Zana and J. Klein, "Segmentation of vessel-like patterns using mathematical morphology and curvature evaluation," IEEE Transactions on Image Processing 10(7), pp. 1010 1019, 2001. 
[12] X. Jiang and D. Mojon, "Adaptive local thresholding by verification-based multithreshold probing with application to vessel detection in retinal images," IEEE Transactions on Pattern Analysis and Machine Intelligence 25(1), pp. 131137, 2003.

[13] S. Chaudhuri, S. Chatterjee, N. Katz, M. Nelson, and M. Goldbaum, "Detection of blood vessels in retinal images using two-dimensional matched filters," IEEE Transactions on Medical Imaging 8(3), pp. 263-269, 1989

[14] Naill Patton, Tariq M. Aslam, Thomas MacGillivray, Ian J. Deary, Baljean Dhillon, Robert H. Eikelboom, Kanagasingam Yogesan, Ian J. Constable, "Retinal image analysis: Concepts, applications and potential", Niall Progress in Retinal and Eye Research 25 (2006) 99-127.

[15] Richard J. Radke, Srinivas Andra, Omar Al-Kofahi, Badrinath Roysam, "Image Change Detection Algorithms:A Systematic Survey", IEEE Transactions on Image Processing, vol. 14, no. 3, march 2005.

[16] A.M. Mendonca and A. Campilho, "Segmentation of retinal blood vessels by combining the detection of centerlines and morphological reconstruction," IEEE Trans. Med. Imag., vol. 25 , no. 9, pp. 1200-1213, Sep. 2006

[17] M. E. Martinez-Perez, A. D. Hughes, S. A. Thom, A. A. Bharath, and K. H. Parker, "Segmentation of blood vessels from red-free and fluorescein retinal images," Med. Image Anal., vol. 11, pp. 47-61, 2007.

[18] Mohammad Saleh Miri and Ali Mahloojifar, "Retinal Image Analysis Using Curvelet Transform and Multistructure Elements Morphology by Reconstruction", IEEE Transactions On Biomedical Engineering, Vol. 58, No. 5, 2011.
[19] M. Kalaivani, M. S. Jeyalakshmi, Aparna.V, "Extraction of Retinal Blood Vessels Using Curvelet Transform and Kirsch's Templates", International Journal of Emerging Technology and Advanced Engineering, Volume 2, Issue $11,2013$.

[20] Farnoosh Ghadiri, Seyed Mohsen Zabihi, Hamid Reza Pourreza, Touka Banaee, “A Novel Method for Vessel Detection Using Contourlet Transform”, 2012.

[21] M.Jenifer Silvia, S.Poovizhi, "Retinal Image Analysis using Ripplet-I Transform and Segmentation using Morphological Gradient”, International Journal of Emerging Technology and Advanced Engineering, Volume 2, Issue 12, 2012.

[22] Parisut Jitpakdee, Pakinee Aimmanee, Bunyarit Uyyanonvara, "A Survey on Hemorrhage Detection in Diabetic Retinopathy Retinal Images", 9th International Conference on Electrical Engineering/Electronics, Computer, Telecommunications and Information Technology (ECTI-CON), 2012.

[23] Sifna N Shajahan, Rajesh Cherian Roy, "An Improved Retinal Blood Vessel Segmentation Algorithm based on Multistructure Elements Morphology", International Journal of Computer Applications (0975 - 8887) Volume 57No.16, November 2012.

[24] Farnoosh Ghadiri , Seyed Mohsen Zabihi , Hamid Reza Pourreza , Touka Banaee, "A Novel Method for Vessel Detection Using Contourlet Transform", Eighteenth National Conference on Communications , 2012-02-03.

[25] T.A.Sangeetha, A.Saradha, "An Efficient Way to Enhance Mammogram Image in Transformation Domain", International Journal of Computer Applications (0975 8887), Volume 60- No.2, December 2012. 